\title{
Early Infant Diagnosis (EID) of HIV: An Experience at a Tertiary Care Hospital in India
}

\author{
Supriya M. Paranjpe, Rajkunwar S. Phakade, Nayana A. Ingole, Preeti R. Mehta
}

Department of Microbiology, Seth GS Medical College \& KEM Hospital, Mumbai, India.

Email: supriyaparanjpe@rediffmail.com

Received November $23^{\text {rd }}, 2011$; revised December $25^{\text {th }}, 2011$; accepted January $10^{\text {th }}, 2012$

\begin{abstract}
Introduction: Early infant diagnosis (EID) confers substantial benefit to HIV infected and HIV uninfected infants and to programmes providing prevention of mother to child transmission (MTCT), but has been challenging to implement in resource limited settings. Objectives: To find out the rate of perinatal transmission in infants born to HIV positive mothers, to study the effect of various predisposing factors on HIV transmission and to evaluate the utility of dried blood spot (DBS) specimen for EID of HIV. Methods: Infants born to HIV positive mothers were tested according to National AIDS Control Organization (NACO) guidelines. Infants of 6 weeks to 6 months of age $(\mathrm{n}=84)$ were diagnosed by DBS PCR; DBS positive results were confirmed by whole blood PCR. Infants $6-18$ months $(\mathrm{n}=47)$ were subjected to antibody test and if positive were confirmed by DNA PCR. Detailed history including type of delivery, single dose nevirapine (SDN) and breast feeding was taken. Results: The HIV transmission rate was $10.69 \%$. In children $\leq 6$ months, who did not receive SDN the positivity was $44.44 \%(4 / 9)$ whereas in those who received SDN it was $6.66 \%(5 / 75),(\mathrm{P}=0.0063)$. In children $>6$ months the positivity rate was significantly higher in breast fed $42.85 \%(3 / 7)$ as compared to non breast fed $5 \%(2 / 40)$ children $(\mathrm{P}=0.0187)$. There was $100 \%$ concordance between DBS and whole blood PCR. Conclusions: In resource limited settings, though HAART should be considered to further reduce MTCT during pregnancy and to prevent the emergence of resistance, SDN should be kept as an option for mothers coming directly in labour. Also, extended ART should be provided to mothers who want to breast feed their children. Early infant diagnosis using DBS specimens will further reduce the morbidity and mortality in these children.
\end{abstract}

Keywords: Early Infant Diagnosis (EID); Dried Blood Spot (DBS); Single Dose Nevirapine (SDN); Mother to Child Transmission of HIV (MTCT)

\section{Introduction}

Since nearly all HIV infections in children are acquired from their mothers, the global epidemiology of HIV in children reflects that of HIV in women. It has been estimated that, in 2008, 1.4 million HIV-infected women gave birth in low and middle income countries and that there were 430,000 new pediatric infections [1]. Without antiretroviral treatment, the risk of transmission of HIV from infected mothers to their children is approximately $15 \%-30 \%$ during pregnancy and labour, with an additional $10 \%-20 \%$ transmission risk attributed to prolonged breast feeding [2].

HIV disease progresses very rapidly in young children, often leading to death.Without care and treatment, one-third of infants die in first year and about a half of children by the second year of life. Asymptomatic children less than 18 months are often missed out on prevention, care, support and treatment [3]. Early diagnosis of HIV infection enables immediate HIV care and initiation of ART as recommended by World Health Organization (WHO). This significantly reduces mortality and hospitalization [4].

Early diagnosis of HIV in infants cannot be accomplished with conventional antibody tests due to passively transferred maternal antibodies for up to 18 months after birth [5].

More precise option for diagnosing infants/children below 18 months is DNA polymerase chain reaction (DNA PCR), which detects HIV-1 pro-viral DNA integrated in the human genome [6-8]. There are two specimens on which DNA PCR can be done namely whole blood sample (WBS) and dried blood spot (DBS). Conventionally whole blood sample has been used. However, recently DBS is being preferred because of problems with transport and storage of WBS.

Significant progress has been made in the global scale-up of prevention of mother-to-child transmission of HIV (PMTCT), in high burden and resource-limited set- 
tings. For the first time, the elimination of MTCT is now considered a realistic public health goal [1].

In India, for Early Infant Diagnosis (EID) of HIV, National AIDS Control Organization, Ministry of Health and Family Welfare, Government of India (NACO) has launched the EID programme [9]. The present study describes the experience and analysis of EID data at a tertiary health care centre. Analysis was carried out to find out the rate of perinatal transmission in infants born to HIV positive mothers, to study the effect of various factors on HIV transmission and to evaluate the utility of DBS specimen for early infant diagnosis of HIV.

\section{Methods}

This study is a retrospective analysis of data obtained by testing infants born to HIV positive mothers at a tertiary care hospital in Mumbai, India from March 2010 to August 2011. The data was analyzed after receiving exemption from review by the institutional ethics committee.

The study included all children 6 weeks to $\leq 18$ months of age born to HIV positive mothers visiting our ICTC (Integrated counseling and Testing Centre, where counseling and HIV testing is done). The NACO guidelines for EID of HIV were followed throughout [9].

As a routine protocol, single dose nevirapine (SDN) is given to HIV positive mothers at the onset of labour and to the child within 72 hours of birth. All such HIV positive mothers were advised to visit the ICTC with their child 6 weeks after delivery. The scheduled visits for children born to HIV positive mothers were at age of 6 weeks, 6 months and 18 months or six weeks after cessation of breast feeding whichever was later.

At their $1^{\text {st }}$ visit, detailed history including date of birth, type of delivery, history of breast feeding and antiretroviral therapy given to the child and mother was noted down. Written informed consent of parent for diagnostic tests of HIV on their child's specimen was taken.

The children were divided in two groups; Group 1 included children up to six months of age whereas Group 2 included children more than six months and up to 18 months of age.

Mothers or children receiving additional anti-retroviral therapy other than SDN and mothers who practiced mixed feeding for their infants were excluded from the study.

In Group 1, blood specimen was collected by a heel/ big toe prick with proper aseptic precautions and dried blood spots (DBS) were made using filter paper (Whatman $903^{\mathrm{TM})}$ ). HIV diagnosis was done by DNA PCR (AMPLICOR HIV-1 DNA PCR) on DBS specimen at a molecular reference laboratory. In case of negative DBS result, the patient was asked to follow up. In case of positive DBS result, whole blood specimen was collected and tested for DNA PCR.

In Group 2, HIV antibody test was done on the serum sample obtained by separation of blood collected by venepuncture. If the result was negative the child was asked to follow up. In case of positive result, DNA PCR was done on the DBS specimen, which if positive was confirmed by a DNA PCR on whole blood sample.

Final diagnosis was made at 18 months of age or 6 weeks after cessation of breast feeding whichever is later by HIV antibody test on serum sample.

Maternal and infant characteristics between transmitters and non transmitters were compared using Chisquare test or Fischer's exact test as applicable.

\section{Results}

The present study included a total of 131 children of which 14 children $(10.69 \%)$ were detected to be HIV positive. The positivity in males was $13.75 \%(11 / 80)$ and in females it was 5.88\% (3/51), $(\mathrm{P}=0.2458)$ (Table 1). 84 children were in Group 1 ( $\leq 6$ months) and 47 were in Group 2 ( $>6$ months and up to 18 months).

In Group 1 , the positivity rate was $10.71 \%(9 / 84)$. The transmission rate in this group of children delivered by vaginal route was $11.67 \%(7 / 60)$ and in those delivered by caesarean section was $8.33 \%(2 / 24)(\mathrm{P}=1.0000)$. In children who received SDN, the positivity was $6.66 \%$ $(5 / 75)$ whereas in those who did not receive SDN it was $44.44 \%(4 / 9)(\mathrm{P}=0.0063)$. Both the groups (who received SDN and not received SDN) matched for breast feeding history (Table 2).

In Group 2, the positivity rate was $10.63 \%(5 / 47)$. In this group, the positivity rate was $42.85 \%(3 / 7)$ in breast fed children and $5 \%(2 / 40)$ in non-breast fed children and the difference was statistically significant $(\mathrm{P}=0.0187)$. Both the groups (who were breast fed and not breast fed) matched for SDN history. Of the 4 mother child pair who received SDN and where the child was HIV infected, 2 children were breastfed (Table 3 ).

All DBS samples which were positive for HIV DNA PCR were also confirmed on WBS. The results were $100 \%$ concordant.

\section{Discussion}

In India, perinatal transmission accounts for $2.4 \%$ of total HIV infection load. Addressing HIV/AIDS in infants and children below 18 months is a significant global challenge. There are dramatic survival benefits for infants started on ART as early as possible. NACO, India has started the EID programme for the early infant diagnosis in 2010 [9].

The present study is an experience of early infant diagnosis in a tertiary care hospital in Mumbai. The rate of 
Table 1. Perinatal transmission in children born to HIV positive mother $(n=131)$.

\begin{tabular}{cccc}
\hline & HIV positive & HIV Negative & P value \\
\hline Male & 11 & 69 & \\
Female & 3 & 48 & 0.2458 \\
Total & 14 & 117 & \\
\hline
\end{tabular}

Table 2. Risk factors for HIV transmission in Group 1 (children $\leq 6$ months) $(n=84)$.

\begin{tabular}{ccccc}
\hline \multirow{2}{*}{ Risk factors } & \multicolumn{4}{c}{ HIV transmission } \\
\cline { 2 - 5 } positive & $\begin{array}{c}\text { HIV } \\
\text { Negative }\end{array}$ & P value \\
\hline \multirow{2}{*}{ Type of delivery } & Vaginal & 7 & 53 & 1.0000 \\
& Caesarean & 2 & 22 & \\
& Received & 5 & 70 & 0.0063 \\
History of SDN & Not received & 4 & 5 & \\
History of feeding & Breast fed & 4 & 17 & 0.2180 \\
& Not breastfed & 5 & 58 & \\
\hline
\end{tabular}

SDN $=$ Single Dose Nevirapine

Table 3. Risk Factors for HIV transmission in Group 2 (children $>6$ months and upto 18 months) $(n=47)$.

\begin{tabular}{ccccc}
\hline \multirow{2}{*}{ Risk factors } & \multicolumn{4}{c}{ HIV transmission } \\
\cline { 2 - 5 } & HIV & HIV & P value \\
& Vaginal & 3 & 33 & 0.5776 \\
Type of delivery & Caesarean & 2 & 9 & \\
& Received & 4 & 40 & 0.2920 \\
History of SDN & Not received & 1 & 2 & \\
& Breast fed & 3 & 4 & 0.0187 \\
History of feeding & Not breastfed & 2 & 38 & \\
\hline
\end{tabular}

SDN $=$ Single Dose Nevirapine.

perinatal transmission in our study was $10.69 \%(14 / 131)$ which is comparable to the other studies in India [10] but is much higher as compared to that reported in the developed countries [2]. The high transmission rate may be attributed to various reasons. Some mothers deliver at home and are not diagnosed during pregnancy and some do not receive SDN as they present after the onset of labour with unknown HIV status. Also, the practice of breast feeding and not receiving ART during breast feeding unless indicated for mother's health contribute to the high positivity.

The WHO 2010 recommendations aim to reduce the risk of MTCT to less than 5\% (or even lower) in breast- feeding populations from a background risk of $35 \%$, and to less than $2 \%$ in non-breastfeeding populations from a background risk of $25 \%$, and will ensure increased maternal and child survival [11]. We still have to go far to achieve this goal.

Various factors are known to affect the vertical transmission of HIV [12]. Caesarean section is believed to decrease the transmission of HIV. In the present study, there was statistically insignificant difference between vaginal deliveries as against Caesarean section deliveries. This maybe because the caesarean sections were not planned surgeries but performed only when obstetrically indicated. Hence, factors such as the timing of the caesarean section in relation to the onset of labour and prolonged rupture of membranes which are known to affect the rate of vertical transmission could not be ruled out. Also, in India delivery by planned caesarean section may not always be feasible in conditions where the delivery takes place at primary health centre or because of cultural factors.

Suppression of maternal viral load to undetectable levels below 50 copies $/ \mathrm{mL}$ using highly active anti-retro-viral therapy (HAART) offers the greatest risk reduction and is currently the standard of care offered in most resource rich countries where MTCT rates have been reduced to $1 \%-2 \%$ [2]. The Zvitambo study clearly supports the initiation of triple ARV prophylaxis among all pregnant women and continuation of the same throughout breast feeding not only to improve maternal health but also to prevent MTCT and to enable safer breast feeding practices [13].

In India, SDN regimen is followed in which single dose of nevirapine is given to mother at the onset of labour and to the new born baby within 72 hours after delivery in all public health facilities [14]. However viral resistance has been detected in HIV infected infants exposed to SDN [15]. In the present study 12 mothers in both groups missed out on SDN as they came in the last stages of labour with unknown HIV status or presented after delivery. SDN was given only to the infants in these cases. In India, often a pregnant woman follows up at one health care facility during her pregnancy and goes to another for her delivery at her native/maternal place. The HIV positivity was significantly higher in these infants in Group 1. Other studies have also highlighted the importance of nevirapine in reducing vertical transmission $[10,16]$. SDN is the simplest regimen and has shown to be feasible and acceptable especially in resource limited settings. Though most researchers now recommend shifting to HAART in pregnancy, SDN should be kept as an option for mothers coming directly in labour.

Breast fed infants have an additional 10\% - 20\% HIV transmission risk. In developed countries, MTCT rates have been brought down to $1 \%-2 \%$ with replacement 
feeding and expanded ART regimens during breast feeding [2]. There are many problems associated with replacement feeding especially in developing countries (such as availability of clean water, insufficient health education and significant cultural barriers). HIV positive mothers also face the dilemma of either giving their babies all the benefits of breastfeeding but exposing them to the risk of HIV infection or avoiding all breastfeeding and increasing the risk of death from diarrhea and malnutrition. In resource limited settings total replacement feeding should be started only when it is acceptable, feasible, affordable, sustainable and safe [10]. As this may not always be possible, it is necessary to think of options which reduce MTCT in breast fed infants.

The current practice of giving SDN to mother and child in India primarily reduces the transmission of virus during delivery but a significant number of infants get infected during breast feeding. In the present study, a significant difference was observed in the HIV positivity of breast fed infants $(42.85 \%)$ as against non breast infants $(5 \%)$ in Group $2(\mathrm{P}=0.0187)$. Chasela C. S. et al. have shown that extended postnatal ART regimen during breast feeding for 28 weeks significantly reduces MTCT [17]. Hence, extended ART should be recommended in HIV positive mothers who want to breastfeed their child and where top feeding is not an option.

The test used to diagnose babies born to HIV infected mothers is HIV DNA PCR which traditionally requires a whole blood sample. Laboratories performing PCR are usually not available in remote places so specimens have to be transported to higher laboratories in urban areas. The WBS requires refrigeration and transport under cold chain maintenance. Continuous supply of electricity is a problem in rural areas so there can be breach in refrigeration and transport is also tedious. In comparison, for DBS, blood sample can easily be coated on filter paper from heel, great toe or finger prick in infants thus avoiding the use of syringes and/or vacutainers. Also the ease of transport of DBS without refrigeration, its property to be biologically stable and less biohazardous makes it a useful tool in resource limited settings. In the present study DBS results were $100 \%$ concordant with WBS results. Other studies also have shown similar findings $[5,10]$. Thus DBS is an attractive alternative to WBS for HIV DNA PCR for EID.

This was the first time that DNA PCR was used to diagnose HIV transmission in infants less than 18 months of age in our setting. All infants diagnosed as HIV infected were immediately started on opportunistic infection prophylaxis and HAART.

To conclude, in resource limited settings, though HAART should be considered to further reduce MTCT during pregnancy and to prevent the emergence of resistance, SDN should be kept as an option for mothers coming directly in labour. Also, extended ART should be provided to mothers who want to breast feed their children. Early infant diagnosis using DBS specimens will further reduce the morbidity and mortality in these children.

\section{Acknowledgements}

The authors are thankful to NACO for initiating and funding the EID program.

\section{REFERENCES}

[1] World Health Organization, "Antiretroviral Drugs for Treating Pregnant Women and Preventing HIV Infection in Infants-Recommendations for a Public Health Approach: 2010 Version," WHO, Geneva, 2010 http://whqlibdoc.who.int/publications/2010/97892415998 18_eng.pdf

[2] J. A. Volmink and B. J. Marais, "HIV: Mother-to-Child Transmission," Clinical Evidence (Online), 2008, Published Online 5 February 2008. http://www.ncbi.nlm.nih.gov/pmc/articles/PMC2907958/

[3] M. L. Newell, H. Coovadia, M. Cortina-Borja, N. Rollins, P. Gaillard and F. Dabis, "Ghent International AIDS Society (IAS) Working Group on HIV Infection in Women and Children. Mortality of Infected and Uninfected Infants Born to HIV-Infected Mothers in Africa: A Pooled Analysis," Lancet, Vol. 364, No. 9441, 2004, pp. 12361243. doi:10.1016/S0140-6736(04)17140-7

[4] World Health Organisation, "WHO Recommendations on the Diagnosis of HIV in Infants and Children, 2010 Version," WHO, Geneva, 2010.

http://whqlibdoc.who.int/publications/2010/97892415990 85_eng.pdf

[5] S. M. Jacob, D. Anitha, R. Vishwanath, S. Parameshwari and N. M. Samuel, "The Use of Dried Blood Spots on Filter Paper for the Diagnosis of HIV-1 in Infants Born to HIV Seropositive Women," Journal of Medical Microbiology, Vol. 26, No. 1, 2008, pp. 71-74. doi:10.4103/0255-0857.38864

[6] G. G. Sherman, P. A. Cooper, A. H. Coovadia, A. J. Puren, S. A. Jones, M. Mokhachane, et al., "Polymerase Chain Reaction for Diagnosis of Human Immunodeficiency Virus Infection in Infancy in Low Resource Settings," Pediatric Infectious Disease Journal, Vol. 24, No. 11, 2005, pp. 993-997.

doi:10.1097/01.inf.0000187036.73539.8d

[7] T. L. Creek, G. G. Sherman, J. Nkengasong, L. Lu, T. Finkbeiner, M. G. Fowler, et al., "Infant Human Immunodeficiency Virus Diagnosis in Resource-Limited Settings: Issues, Technologies, and Country Experiences," American Journal of Obstetrics \& Gynecology, Vol. 197, No. 3, Supplement, 2007, pp. S64-S71. doi:10.1016/j.ajog.2007.03.002

[8] W. Stevens, G. Sherman, R. Downing, L. M. Parsons, C. Y. Ou, S. Crowley, et al., "Role of the Laboratory in Ensuring Global Access to ARV Treatment for HIV-Infected 
Children: Consensus Statement on the Performance of Laboratory Assays for Early Infant Diagnosis," Open Chemical Physics Journal, Vol. 2, 2008, pp. 17-25. doi: $10.2174 / 1874613600802010017$

[9] National AIDS Control Organization, "Guidelines for Care of HIV Exposed Infants and Children Less than 18 Months 2010 Jan," National AIDS Control Organisation, India. http://www.nacoonline.org/upload/Care\%20\&\%20Treatm ent/Monograph\%20on\%20HIV_CST\% 20 division\%20_ 21st\%20June\%202010.pdf

[10] K. K. Jain, R. K. Mahajan, M. Shevkani and P. Kumar, "Early Infant Diagnosis: A New Tool of HIV Diagnosis in Children," Journal of Community Medicine, Vol. 36, No. 2, 2011, pp. 139-142. doi:10.4103/0970-0218.84134

[11] World Health Organisation, "New Guidance on Prevention of Mother-to-Child Transmission of HIV And Infant Feeding in the Context of HIV," WHO, Geneva, 2010. http://www.who.int/hiv/pub/mtct/PMTCTfactsheet/en/ind ex.html

[12] J. P. McGowan and S. S. Shah, "Prevention of Perinatal HIV Transmission During Pregnancy," Journal of Antimicrobial Chemotherapy, Vol. 46, No. 5, 2000, pp. 657-668. doi: $10.1093 / \mathrm{jac} / 46.5 .657$

[13] J. W. Hargrove and J. H. Humphrey, "ZVITAMBO Study Group. Mortality among HIV-Positive Postpartum Women with High CD4 Cell Counts in Zimbabwe," AIDS, Vol. 24, No. 3, 2010, pp. F11-F14. doi:10.1097/QAD.0b013e328335749d

[14] National AIDS Control Organization, "Prevention of Parent to Child Transmission of HIV," National AIDS Control Organisation, India.

http://www.nacoonline.org/National_AIDS_Control_Prog $\mathrm{ram} /$ Services_for_Prevention/PPTCT/

[15] M. Lallemant and G. Jourdain, "Preventing Mother-toChild Transmission of HIV-Protecting This Generation and the Next," New England Journal of Medicine, Vol. 363, No. 16, 2010, pp. 1570-1572. doi:10.1056/NEJMe1009863

[16] A. Gupta, N. Gupte, J. Sastry, K. E. Bharucha, R. Bhosale, P. Kulkarni, et al., "BJMC-JHU MIT Study Team. Mother-to-Child Transmission of HIV among Women Who Chose Not to Exclusively Breastfeed Their Infants in Pune, India," Indian Journal of Medical Research, Vol. 126, No. 2, 2007, pp. 131-134.

[17] C. S. Chasela, M. G. Hudgens, D. J. Jamieso, D. Kayira, M. C. Hosseinipour, A. P. Kourtis, et al., "BAN Study Group. Maternal or Infant Antiretroviral Drugs to Reduce HIV-1 Transmission," New England Journal of Medicine, Vol. 362, No. 24, 2010, pp. 2271-2281. doi:10.1056/NEJMoa0911486 